\section{Manchmal kommt noch was nach}

\section{Kommt es nach einer Allergeninjektion im Rahmen einer Hyposensi- bilisierung zu einer systemischen Reaktion, so kann diese einige Stunden später erneut aufflammen. Wie häufig solche biphasichen Verläufe sind, wollten Ärzte der US-Armee herausfinden.}

D er Anteil anphylaktischer Reaktionen, bei denen es zu einem biphasischen Verlauf kommt, wird in der Literatur mit 1 bis $20 \%$ angegeben. Nach einer Allergeninjektion im Rahmen einer spezifischen Immuntherapie (SIT) wurde überhaupt erst viermal über eine biphasische Reaktion berichtet. Um der tatsächlichen Inzidenz und möglichen Risikofaktoren auf den Grund zu gehen, untersuchten Ärzte verschiedener USMilitärkrankenhäuser prospektiv alle $\mathrm{Pa}$ tienten, die aufgrund einer systemischen Reaktion nach einer SIT Adrenalin erhalten hatten. 55 Patienten entwickelten insgesamt 60 systemische Reaktionen, davon waren 14 biphasisch - also fast jede vierte. Acht der Reaktionenen manifestierten sich extrakutan, sechs ausschließlich kutan.

Die Analyse möglicher Einflussfaktoren zeigte eine signifikante Korrelation zwischen biphasischer Reaktion und weiblichem Geschlecht $(\mathrm{p}=0,03)$, höherem Lebensalter $(p=0,01)$ und der Gabe von mehr als einer Adrenalindosis bei der ersten Reaktion ( $p=0,001)$. Kei-

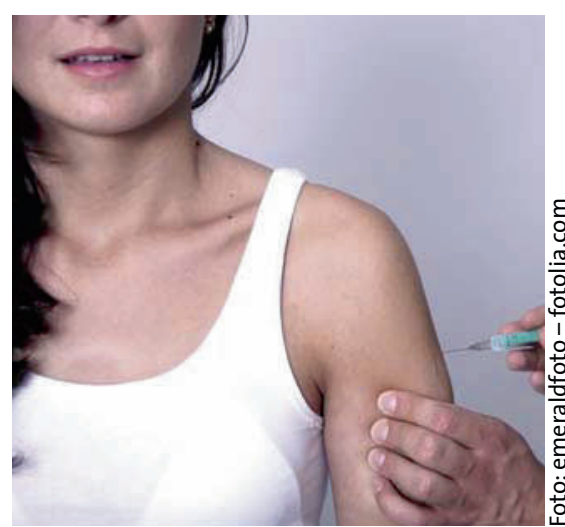

Frauen entwickeln nach einer Allergeninjektion häufiger biphasische Reaktionen als Männer.

\title{
Ein Gras ist genug
}

Es ist umstritten, ob die spezifische Immuntherapie bei Patienten mit einer Gräserpollenallergie, besser mit einer Gräsermischung erfolgen sollte oder ob ein Monoextrakt auch ausreicht. Eine aktuelle In-vitroUntersuchung belegt eine hohe Kreuzreaktivität zwischen verschiedenen Vertretern der Süßgräser-Unterfamilie Pooideae.

$\mathrm{E}^{\mathrm{i}}$ in dänisch-deutsches Forscherteam griff zunächst auf Seren von Gräserpollen-sensibilisierten Probanden zurück, die sich noch keiner Hyposensibilisierung unterzogen hatten. Sowohl mittels direkter Bindung im Immunoassay als auch mittels Inhibitionsstudien konnte gezeigt werden, dass das spezifische IgE aus diesen Seren mit vier Mischextrakten, die fünf bis zehn Pooideae-Spezies enthielten, und einem reinen Phleum-pratense-Extrakt in hohem $\mathrm{Maß}$ kreuzreagierte. Um zu überprüfen, ob verschiedene Extrakte unterschied- liche T-Zell-Reaktionen auslösen, untersuchten die Forscher zudem für sechs Monoextrakte die Stimulation von TZellen von Probanden, die spezifisch auf Phl p 1 und/oder Phl p 5 reagierten. Fünf Extrakte lösten eine vergleichbare T-Zell-Proliferation aus. Nur beim Ruchgrasextrakt (Anthoxanthum odoratum), der den geringsten Gehalt an Gruppe-1/5-Allergenen aufwies, fiel die Reaktion schwächer aus.

Außerdem überprüften die Forscher bei Patienten, die über 28 Tage eine sublinguale oder über ein bis drei Jahre eine nen Einfluss hatten dagegen die Art der Immuntherapie (Aeroallergene oder Insektengift), ein bestehendes Asthma bronchiale oder eine Therapie mit Antihistaminika. Auch die Ausprägung der initialen Symptome, die Zeit bis zum Auftreten der ersten Reaktion, die Dauer bis zur ersten Adrenalininjektion oder das Ansprechen auf diese Ininjektion spielten für die Wahrscheinlichkeit einer biphasischen Reaktion keine Rolle.

Trat eine zweite Reaktion auf, war diese signifikant schwächer ausgeprägt als die erste, eine erneute Adrenalingabe war in keinem Fall nötig.

Fazit: Entwickeln Patienten nach einer Injektion im Rahmen einer Hyposensibilisierung eine systemische Reaktion, die eine Adrenalingabe nötig macht, so ist ein biphasischer Verlauf durchaus häufig: $23 \%$ der Patienten dieser Kohorte erlitten eine zweite Reaktion. Diese verlief allerdings eher mild und war selbstlimititiert.

Scranton SE et al. Incidence and characteristics of biphasic reactions after allergen immunotherapy. J Allergy Clin Immunol 2009; 123: 493-8

subkutane spezifische Immuntherapie mit einem Phleum-pratense-Monoextrakt erhalten hatten, ob die durch die Therapie induzierten spezifischen $\mathrm{IgG}_{4}$-Antikörper mit Allergenen anderer PooideaeSpezies kreuzreagierten. Tatsächlich korrelierten die $\mathrm{IgG}_{4}$-Antikörper-Reaktionen im Immunoassay auf acht Monound vier Mischextrakte mit den Reaktionen auf einen Phleum-pratense-Monoextrakt.

Fazit: Die T- und B-Zell-Antworten gegenüber verschiedenen Pooideae-Spezies weisen ein hohes Maß an Kreuzreaktivität auf. Eine Hyposensibilisierung mit nur einer Spezies sollte daher ähnlich wirksam sein wie mit einem Mischextrakt.

pe

Hejl C et al. Phleum pratense alone is sufficient for allergen-specific immunotherapy against allergy to Pooideae grass pollens. Clin Exp Allergy 2009; 39: 752-9 